\title{
Data accuracy and completeness of monthly midwifery returns indicators of Ejisu Juaben Health Directorate of Ghana
}

\author{
Richard Okyere Boadu ${ }^{1}$, Peter Agyei-Baffour ${ }^{2}$, Anthony Kwaku Edusei ${ }^{3}$ \\ ${ }^{1}$ Department of Health Information Management School of Allied Health Sciences, College of Health and Allied \\ Health Sciences, University of Cape Coast, Ghana \\ ${ }^{2,3}$ School of Public Health, Kwame Nkrumah University of Science and Technology, Ghana
}

\begin{tabular}{l} 
Article Info \\
\hline Article history: \\
Received Oct 10, 2018 \\
Revised Nov 02, 2018 \\
Accepted Dec 05, 2018 \\
\hline
\end{tabular}

\section{Keywords:}

Data accuracy

Data completeness

Quality improvement process

Routine health information

\begin{abstract}
The broad range of activities contained in the provision of Primary Health Care (PHC) places a burden on providers to make optimal use of limited resources to achieve maximal health benefit to the population served. All too often, ad hoc decisions and personal preferences guide PHC resource allocations, making accountability for results impossible. Problems constraining Routine Health Information System (RHIS) performance in low-income countries include: poor data quality; limited use of available information; weaknesses in how data are analyzed and poor RHIS management practices. This study sought to investigate these constraints. A non-experimental before and after study involving baseline assessment of data accuracy and completeness, application of innovative strategies such as mentoring and coaching of Health Information Officers in data quality improvement process. Coincidentally, the intervention improved both data accuracy and completeness performance significantly among the participating facilities. The outstanding performance may be attributed to management's new orientation and growing interest towards quality data. Engaging frontline staff in data quality improvement work and provision of regular feedback leads to improvement in data accuracy and completeness. This has implications for decision-making and resource allocation, especially in low-income countries, where the routine health information management system relies heavily on paper work.
\end{abstract}

Copyright $\odot 2019$ Institute of Advanced Engineering and Science. All rights reserved.

Corresponding Author:

Richard Okyere Boadu,

Department of Health Information Management,

School of Allied Health Sciences, College of Health and Allied Health Sciences,

University of Cape Coast, Cape Coast, Ghana, Cell: +233 244227592.

Email: richard.boadu@ucc.edu.gh

\section{INTRODUCTION}

The all-encompassing range of activities contained in the provision of Primary Health Care (PHC) places a burden on health service providers to make efficient use of scarce resources to achieve maximal and effective health outcomes in the community. In many instances, ad hoc decisions and personal preferences guide PHC resource allocations due to lack of confidence in the quality of Routine Health Information System (RHIS). As a result, impact assessment of public health interventions and accountability for results become difficult or impossible to establish. Problems constraining RHIS performance in low-income countries include: poor data quality; limited use of available information; weaknesses in how data are analyzed and poor RHIS management practices [1-3].

It is common to observe that data generated by health care providers in the health facilities and at community outreach services have gaps either left unfilled on the pretence of lack of sufficient time due to work overload, lack of appropriate registers or due to lack of appreciation of the danger in using incomplete data in decision making. There is wide recognition of the function of data to underpin decision-making. Data 
processed from health information into biostatistics are used to improve overall management and performance of the health sector. For example, the Ministry of Health (MOH) and Ghana Health Service (GHS) have a formal performance agreement in which the GHS is engaged to deliver specific outputs and outcomes using the inputs provided by $\mathrm{MOH}$; as manifested in monthly or quarterly reporting based on performance indicators in the contracts signed. Usually, the agreement presupposes that systems to provide reliable and timely information are in place. In reality however, this is not the case. The degree of understanding of the role and function of health information is illustrated in the Bulletin of Health Information published by the Ministry of Health [4]. This draws attention to the need to transform data into information for action, especially at the local level.

Demand for data in response to changing health partner funding mechanisms has become very significant. There has been the need to show more precisely the commensurate achievements and benefits to vulnerable groups in particular based on partner investments in the health sector. Countries and international organizations have recently renewed their interest in the performance of the health systems. This has led to the development of performance indicators for monitoring, assessing, and managing health systems to achieve effectiveness, equity, efficiency and quality in healthcare delivery [5-6].

Mechanisms to address the low understanding and unreliably and untimely information include facilitative supervision, on-the-job training, bi-annual and annual performance hearing [7]. The annual district, regional and national performance hearings instituted by Ghana Health Service serve as a peer review mechanism and are used to validate the reported performance figures. Despite these significant efforts, significant data quality issues and their application remain poor at the regional and district levels and poorest at the sub-district and community levels. Previous HMIS Assessment [8] in Ghana revealed the lack of a clear policy and legal framework for health information and health data reporting which has contributed to the incomplete reporting in the health sector and the lack of data management structures within the private health sector.

Data quality is described in four dimensions: relevance, completeness, timeliness and accuracy [9-11]. Relevance is assessed by comparing data collected against management information needs. Completeness is measured not only as filling in all data elements in the facility report form, but also as the proportion of facilities reporting in an administrative area (e.g. province or district). In Ghana, the health facilities are supposed to submit data to the next level by the 5th of ensuing months. At the district level, data submitted by the health facilities are supposed to be entered into the District Health Information Management System (DHIMS2) by 15th of the ensuing month. Timeliness is assessed as submission of the reports by an accepted deadline. Accuracy is measured by comparing data between facility records and reports, and between facility reports and administrative area databases, respectively.

The interpretation of comparative routine data thus faces three major challenges: (a) The need for appropriate measures. (b) The need to control for case mix and other variables. (c) The need to minimize chance variability. These are all underpinned by a fourth challenge - namely, the need for high quality data. It is self-evident that poor quality data compound the inherent problems of interpretation of routine data described above and can undermine even the most sophisticated quality assessment tool [12]. It is well recognized that no data are completely accurate. The actual concern with data quality is to ensure not that the data are perfect, but that they are accurate enough, timely enough, and consistent enough for the organization to use it for appropriate and reliable decision-making [13].

Nevertheless, even in well resourced, well organized research studies, it is difficult to ensure that data are complete and of a consistently high quality [14]. It is challenging still to ensure that routine data are of a high quality; the many factors that can compromise the reliability of both primary and secondary data have been well-defined [15]. For instance, those collecting and entering routine health data at the health facility are likely to be doing so as one of many daily tasks: data collection may be in conflict with other priorities and limited understanding of the purpose of the data may lead to unwitting errors in non-standard situations. Many different individuals will be involved in data collection and recording over long time periods and the risks of mistakes, inconsistencies or gaps are high [12]. Thus, when routine data are used for quality assessment purposes, it is a common finding that they are inaccurate, with omissions and erroneous inclusions, or incomplete (especially in relation to some treatments) or insufficiently detailed for the purpose [16].

The data quality challenges identified in the previous assessments appears not to be different from what pertain in the Ejisu Juaben Municipal Routine Health Information System. The Municipal RHIS is characterized by data incompleteness, inaccuracy and timeliness which further raise doubts on whether decision making at the various levels of health service delivery reflects what exists on the ground. The 2010 annual report on performance review of the Ejisu-Juaben Municipality revealed that, about $60 \%$ of health facilities within the municipal which are either privately owned or under Christian Health Association of Ghana (CHAG), do not submit their data on their health services delivery to the Municipal Health

Data accuracy and completeness of monthly midwifery returns indicators in... (Richard Okyere Boadu) 
Directorate for aggregation. This implies that health planning and decision making in the municipality are based on only $40 \%$ of the quantum of health facilities in the public facilities. But the level of quality of data from this $40 \%$ remains an illusion. Undoubtedly, this is likely to affect the quality and content of data and decision made with it. This study sought to improve data completeness and accuracy in Ejisu Juaben Municipal Health Directorate.

\section{RESEARCH METHOD}

This section presents the methods and procedures used in the study. The section details profile of the study area, study population, sample size, sample technique, data collection techniques and tools. It also presents measurements and data analysis, ethical considerations, assumptions of the study as well as reliability and validity of data collection process.

\subsection{Study design}

A non-experimental pretest-posttest design involving baseline assessment of data completeness and accuracy, application of quality improvement interventions such as mentoring and coaching of District Information Officers in data quality improvement process, facility-based quarterly data reconstruction, monthly feedback to frontline providers, use of provisional registers and tally sheets for documenting outreach and static services, and frontline staff training and orientation on monthly midwifery returns indicators definition at the facility level.

\subsection{Profile of study area}

The study was carried out in the Ejisu Juaben Municipality in the Ashanti Region. Ejisu Juaben Municipal is one of the 27 districts and municipals in Ashanti Region. It is located in the South-Eastern part of the Region and shares boundaries with Kwabre, Afigya-Sekyere, Sekyere East and West to the North: Asante Akim North and South Municipal to the East, Bosomtwe District to the South and Kumasi Metropolis to the West. The municipal has a projected population of 146,762 based on 2010 population and housing census with growth rate of $3.4 \%$ per annum (Population and Housing Census, Ghana Statistical Service, 2010). There are 91 communities. The road network is fairly good with few tarred roads. The rest are mainly feeder roads, some of which are not motorable especially during the rainy season. Some of the communities are extremely hard to reach during the rainy season. The only means by which these communities could be reached is by boat, swimming and walking. For the purpose of Health Administration, the municipal has been divided into five sub-municipals namely; Achiase, Bomfa, Ejisu, Juaben and Kwaso. All the communities within the municipal have Community-Based Surveillance Volunteers (CBSVs). The total number of CBSVs is 200 . The total staff strength is 247 . There are Twenty-Six health facilities with eighty-one outreach points.

\subsection{Study population}

The study population involved health staff and management who collect or use data routinely in all health facilities: both private and public-owned, in the Ejisu Juaben Municipality. About $65 \%$ of the staff are females whiles $35 \%$ are males. Majority of the staff had average working experience of 4.8 years. In all the facilities, staff who had worked for at least 6 months are involved in data generation, processing and use, and consented to participate were included. Potential participants who did not meet the inclusion criteria were excluded.

\subsection{Sample size}

Purposive sampling was used to recruit all 26 health facilities in the Municipality. However, only 20 consented to participate. Thus, all analysis and conclusions are based on the 20 and not 26.

\subsection{Recruitment process}

The selected participated facilities were then taken through an informed consenting process and those who voluntarily consented were enrolled. Total number of 151 out of 247 health staffs was recruited into the study. However, only 141 consented to participate.

\subsection{Data collection techniques and tools}

Data were obtained from health facilities in Ejisu-Juaben Municipality. The survey instruments used were adapted from those in the Performance of Routine Information System Management (PRISM) tool package [17-18] Uganda [19-20] and further refined in China [21]. 


\subsection{RHIS performance diagnostic tool}

The diagnostic tool was used. The diagnostic tool measures strengths and weaknesses in different dimensions of data quality, information use, and RHIS processes. The tool provides information about technical determinants such as perceived user friendliness of forms, software, and RHIS design, and quality of supervisory visits. The diagnostic tool consists of four forms on data quality and information use: two for the district level or higher and two for the facility level. These forms differ by level because some determinants such as timeliness and completeness could not be measured at the facility level. Similarly, the questions related to RHIS design and information technology are more relevant for the district or higher level. However, the information use section is almost similar for both levels, except for the addition of a section on the quality of supervision at the facility level.

\subsection{Limitations of data collection tools}

The data quality and information use indicators are not all inclusive. To improve its utility, the diagnostic tool should be adapted to meet the needs of the RHIS in a given country to reflect their particular objectives and data processes. Before implementing the adapted questionnaires, pre testing is needed to fine tune it to make it more suitable.

\subsection{Measurements and data analysis}

Data accuracy and completeness assessment required quarterly visits to the health facilities to meet with the health providers and review their primary sources of data which contain records of services provided to clients. The operational definition of data accuracy is congruence between reconstructed and reported data for a specific indicator in a specific time period. In order to assess data accuracy, two sets of data on each indicator are required: reconstructed data from the primary source registers at the point of care and reported data submitted to the district health office. Contact person at the facility and the District Health Information Officer (DHIO) do independent reconstruction on each selected indicator from the appropriate register, which is the primary source document, and reconcile all discrepancies as gold standard for that month. The DHIO compares the gold standard figure with reported figure for the same period of assessment.

The percentage data accuracy of specific indicator was used to determine longitudinal data accuracy. The percentage of accuracy is calculated by dividing reported data by reconstructed data (i.e. gold standard) and multiplying by 100 (i.e. Reported/Reconstructed X 100). If the result is:

a. Equal to $100 \%$, then reported and reconstructed values are the same

b. Less than $100 \%$, then reconstructed value is higher, which means data submitted was under reported

c. Greater than $100 \%$, then reconstructed value is lower, which means data submitted was over reported.

Note that if either value is 0 , the data are not used in calculating accuracy.

To assess data accuracy at Municipal level, the study calculates the average data accuracy percentages for all facilities each month (i.e. add up all the data accuracy percentages for each month and divide by the number of facilities) and plot on a line graph, as seen on Figure1 for the purpose of this study data completeness means that health facilities are submitting completed summary sheets to the Municipal Health Management Team (MHMT) every month and that all required data elements are filled. To keep track of improvement over time, a control chart was used to plot percentage of data completeness before and after intervention. To assess data completeness on a Municipal level, the study calculates the average data completeness percentages for all facilities each month (i.e. add up all the data completeness percentages for each month and divide by the number of sub-facilities) and plot on a line graph, as seen on Figure 2.

To determine how accurate, the data is at particular facility during the baseline and end line assessments, the study first determines which data points or elements from the monthly reports need to be checked. There are only four data points per month that can be captured because the survey tool does not allow time for more. The data points were chosen from different sections of the monthly report, in order to reflect on the performance of different health services and their associated support mechanisms. The same data point or elements were tracked for both baseline and end line in order to assess if there has been improvement after the intervention.

\subsection{Innovations}

Mentoring and coaching of Health Information Officers in data quality improvement process, quarterly data reconstruction at the facility level, monthly feedback from data quality team to frontline providers. Other innovations tested included the use of provisional registers and tally sheets for outreach and community static services to address the complain of service providers not able to carry along bulky registers. With the old practice, service providers either documented outreach services on piece of paper with the intension of transferring them into the official registers later. Many times, the providers forget updating 
the register before completing the monthly report. Other change ideas implemented include training and orientation on monthly midwifery returns indicators definition at the facility level.

\subsection{Control chart}

Control chart was used to perform time series analysis for monitoring monthly changes in data quality improvement in some selected key monthly midwifery returns indicators. The control charts, also known as Shewhart charts or process-behavior charts, in statistical process control are tools used to determine whether or not a process, in this case, data accuracy and completeness process, is in a state of statistical control. For instance, the control chart was used to monitor performance of percentage of data accuracy in Antenatal care (ANC) registrants by comparing reported figures with that of the register overtime, as indicated in figures 1. Data accuracy is given by the difference between reported figures and reconstructed figures in the register multiply by $100 \%$. If reported figure equals that of the register, then we have $100 \%$ accuracy. On the other hand, if percentage of accuracy exceeds $100 \%$ then we have over reporting. Under reporting occurs when percentage of accuracy is below 100\%. Data completeness means that health facilities are submitting each required summary sheet to the Municipal Health Management Team (MHMT) every month and that all required data elements are completed on Midwife Returns Forms submitted. For instance, control chart used to monitor improvement in percentage of completed midwifery return forms over a period.

\subsection{Ethical considerations}

The study protocol was submitted to the Committee for Human Research Publications and Ethics (CHRPE) of Kwame Nkrumah University of Science and Technology for clearance. Permission was also sought from the Municipal Health Directorate to implement the study. The research team was introduced to all the municipal and Sub-municipal health facilities by the Municipal Director of Health. The selected facilities were briefed about the study's objectives, potential risks, benefits, the role of the facilities and their freedom to participate and withdraw at any stage of the study. Information leaflets were distributed to potential participants to read and ask questions or make comments. Facilities that agreed to participate were asked to sign an informed consent form to confirm their willingness to be part of the study. The contact address of the Research Team was given to participating facilities for future contacts if they so wished.

\subsection{Assumptions of study}

This study assumed that once facility staff are trained in data quality improvement protocol they would apply the principles continuously to improve their data and also use the improved data for planning and decision-making. Data quality improvement team in the municipal would embark on quarterly data quality assessment at the facility level. Resources such as motorbike/vehicle or fuel would be made available for quality improvement team for field visits on quarterly basis. The study further assumed that data quality improvement issues would be incorporated into the municipal health directorate weekly meetings

\subsection{Reliability and validity}

Questionnaires for the study were pre-tested at Mampong in Sekyere West Municipality, which is not part of the study area but has common characteristics with Ejisu-Juaben Municipality. Based on feedback from the field pre-test, the tools were modified to ensure its suitability for the study. Research Assistants were given 3-day training on the research protocol and data collection tools. This was important to make them familiar with the tools and the expected way of questionnaire administration to reduce inconsistencies and biases. To ensure data quality, data verification was conducted for randomly selected administered questionnaires. Also, data validation checks were included in the data entry software to minimize data entry errors. Data quality assessment protocol was used to assess data quality to ensure reliable procedure for data quality improvement at the health facilities.

\section{RESULTS AND ANALYSIS}

The following are the results from the baseline and post intervention involving 20 health facilities in Ejisu Juaben Municipality. For instance, Table 3 revealed that there has been enormous improvement in support supervision and feedback mechanism from municipal health supervisors to the frontline providers at the facility level. This triggered an improvement in data completeness and accuracy as shown in Figures 1, and 2. Consequently, there has been improvement in data use at the facility among health managers for planning and decision-making. 
This section presents also discussion of the results in line with literature. It further analyses the implications of the findings in relation to similar work or research done by others, highlighting similarities and differences and their implications on health service delivery, especially in Ghana.

\subsection{Data accuracy check}

Data accuracy checks were conducted by matching two months reported figures of some maternal health indicators (e.g. ANC registrants, $1^{\text {st }}$ trimester registrants, $4^{\text {th }}$ ANC visits, IPT3) with that of the Antenatal care registers. Table 1 shows the performance of data accuracy during baseline and end line assessment.

Table 1. Performance of data accuracy

\begin{tabular}{lcccc}
\hline \multicolumn{1}{c}{ Data Accuracy Check } & \multicolumn{2}{c}{ Baseline } & $(2012)$ & \multicolumn{2}{c}{ Endline (2014) } \\
& Yes & No & Yes & No \\
\hline $\begin{array}{l}\text { Number of facilities that had their 2 months reported } \\
\text { figures in monthly midwifery returns (e.g. ANC }\end{array}$ & 3 & 17 & 18 & 2 \\
registrants, $1^{\text {st }}$ trimester registrants, $4^{\text {th }}$ ANC visits, & $(15 \%)$ & $(85 \%)$ & $(90 \%)$ & $(10 \%)$ \\
IPT3) matching the figures in the ANC register. & & & \\
Did you receive a directive from the Senior Management/ & & & \\
municipal/sub-municipal office to: & 3 & 17 & 18 & 2 \\
Check the data accuracy at least once in three & $(15 \%)$ & $(85 \%)$ & $(90 \%)$ & $(10 \%)$ \\
months? & 2 & 18 & 17 & 3 \\
Fill the monthly/quarterly report form completely & $(10 \%)$ & $(90 \%)$ & $(85 \%)$ & $(15 \%)$ \\
& 10 & 10 & 20 & 0 \\
Submit report by declared deadline & $(50 \%)$ & $(50 \%)$ & $(100 \%)$ & $(0 \%)$ \\
& & & \\
Did you receive a directive from the Senior Management// & & & 0 \\
municipal/sub-municipal office that there will be consequences: & 0 & 20 & 20 & 0 \\
if you do not check the data accuracy & $(0 \%)$ & $(100 \%)$ & $(100 \%)$ & $(0 \%)$ \\
& 0 & 20 & 20 & 0 \\
If you do not fill the monthly reporting form & $(0 \%)$ & $(100 \%)$ & $(100 \%)$ & $(0 \%)$ \\
completely & 8 & 12 & 20 & 0 \\
If you do not submit the monthly report by declared & $(40 \%)$ & $(60 \%)$ & $(100 \%)$ & $(0 \%)$ \\
deadline & 4 & 16 & 19 & 1 \\
Overall Data Accuracy Check Performance & $(20 \%)$ & $(80 \%)$ & $(95 \%)$ & $(5 \%)$ \\
\hline
\end{tabular}

The overall data accuracy performance, 4 out of 20 facilities in baseline performed data accuracy check compared to 19 out of 20 in end line, showing significant improvement as showm in Table 1 . It is seen that besides innovative strategies implemented by the study; involvement of senior management in giving directives to the facilities with consequences of data inaccuracy check. For instance, while none of the participating facilities received directive from senior management to check data accuracy and completeness in baseline survey, the end line result suggests that all the 20 facilities received such instructions prior to end line assessment as shown in Table 1. This achievement could be attributed to management's new orientation and growing interest towards quality data.

\subsection{Data completeness}

Table 2 shows the performance of data completeness at the baseline and end line assessment. As shown in Table 2, the overall data completeness performance indicates that 4 out of 20 facilities submitted completed monthly report in baseline compared with 19 out of 20 facilities in end line.

Table 2. Performance of data completeness

\begin{tabular}{|c|c|c|c|c|}
\hline \multirow{2}{*}{ Data Completeness } & \multicolumn{2}{|c|}{ Baseline (2012) } & \multicolumn{2}{|c|}{ Endline (2014) } \\
\hline & Yes & No & Yes & No \\
\hline $\begin{array}{l}\text { Number of facilities that reported the number } \\
\text { of data items in the RHIS monthly report that } \\
\text { facility need to report in the last two months } \\
\text { (this excludes the number of data items for } \\
\text { services not provided by this health facility). }\end{array}$ & $\begin{array}{c}8 \\
(40)\end{array}$ & $\begin{array}{c}12 \\
(60 \%)\end{array}$ & $\begin{array}{c}20 \\
(100 \%)\end{array}$ & $\begin{array}{c}0 \\
(0 \%)\end{array}$ \\
\hline $\begin{array}{l}\text { Number of facilities that filled all monthly } \\
\text { report without leaving any blank space in at } \\
\text { least one of the reports. }\end{array}$ & $\begin{array}{c}0 \\
(0 \%)\end{array}$ & $\begin{array}{c}20 \\
(100 \%)\end{array}$ & $\begin{array}{c}18 \\
(90 \%)\end{array}$ & $\begin{array}{c}2 \\
(10 \%)\end{array}$ \\
\hline Overall Data Completeness Performance & $\begin{array}{c}4 \\
(20)\end{array}$ & $\begin{array}{c}16 \\
(80 \%)\end{array}$ & $\begin{array}{c}19 \\
(95 \%)\end{array}$ & $\begin{array}{c}1 \\
(5 \%)\end{array}$ \\
\hline
\end{tabular}


However, 8 out 20 and 20 out of 20 facilities respectively in baseline and end line reported the required number of monthly report that the facilities needed to report in the last two months prior to the assessment (this excludes the number of data items for services not provided by this health facility). However, in terms of filling out all monthly reports without leaving any blank space in at least one of the reports, none of the facilities achieved this in baseline whereas 18 out of 20 facilities were able to achieve it in end line as portrayed in Table 2.

\subsection{Data quality improvement (accuracy and completeness)}

Figures 1 and 2 are control charts, also known as Shewhart charts or process-behavior charts, in statistical process control are tools used to determine whether or not a business process, in this case, data accuracy and data completeness improvement overtime, is in a state of statistical control. For instance, the control chart was used to monitor performance of percentage of ANC data accuracy by comparing reported figures with that of the register is shown in Figure 1 as well as data completeness of monthly midwifery returns as shown in Figure 2 overtime.

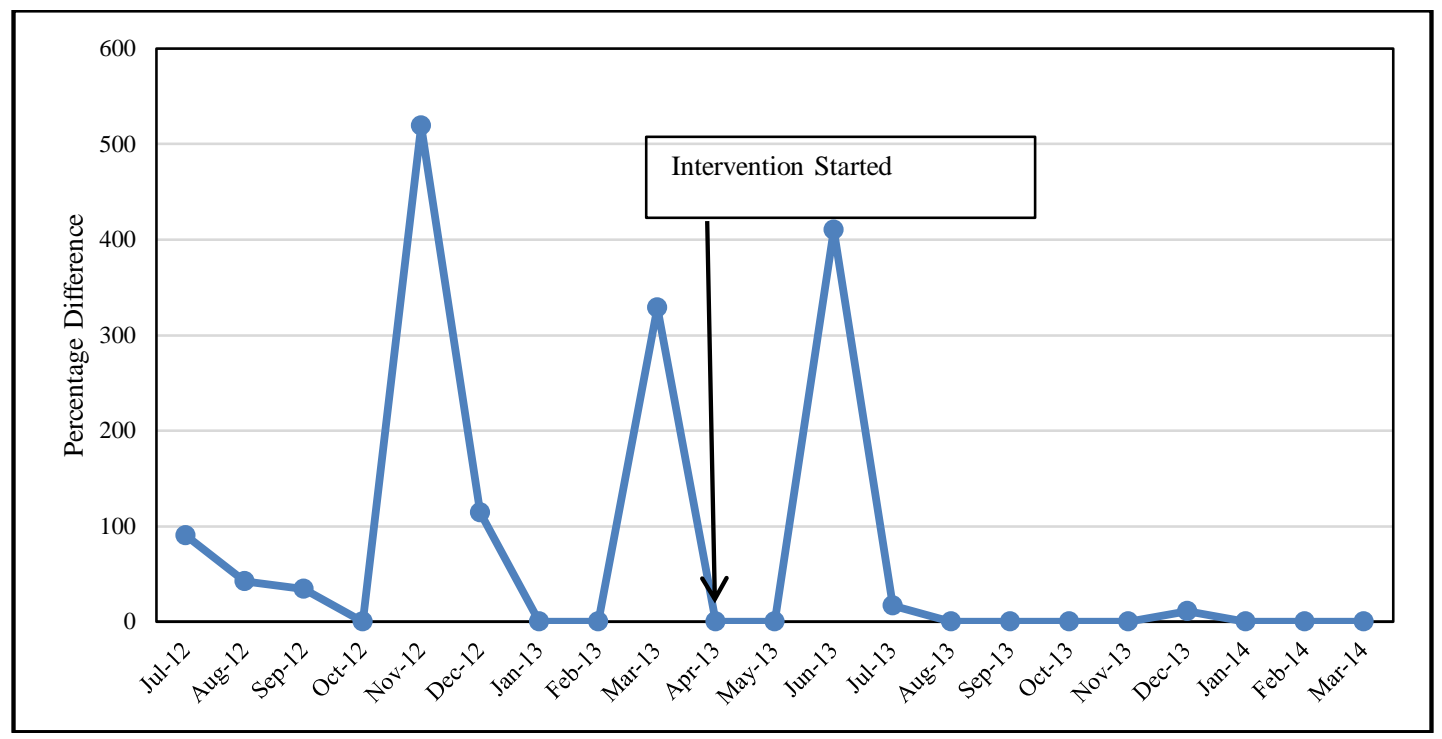

Figure 1. Percentage difference in reported \& reconstructed ANC registrants data values-Ejisu Juaben municipality (July 2012-March 2014)

Antenatal care visits are times when women benefit from comprehensive service package including prevention and control of anemia, malaria, tetanus, and screening/management of conditions that can affect their health and subsequent growth and survival of their unborn babies. They also receive counseling on a range of issues including nutrition, birth preparedness and complication readiness [22]. Antenatal registrant's indicator was found to be one of the problematic erroneous data elements in the Ejisu Juaben health directorate. Data quality was unpredictably over reported (not less than four times the actual value) during the period of July 2012 to August 2013 as shown in Figure 1. While data capture especially from peripheral levels of the service and the private sector could be responsible in the poor quality of antenatal data. Though intervention started in April 2013, the Municipal Health Directorate experienced data accuracy improvement after three months; and sustain the gain till the end of the project.

Data completeness appeared improving from $87 \%$ to $95 \%$ before intervention and drop to $9 \%$ as shown in Figure 2. However, in July 2013 the directorate recorded 100\% data completeness and sustains the gain up to the end of the project. 


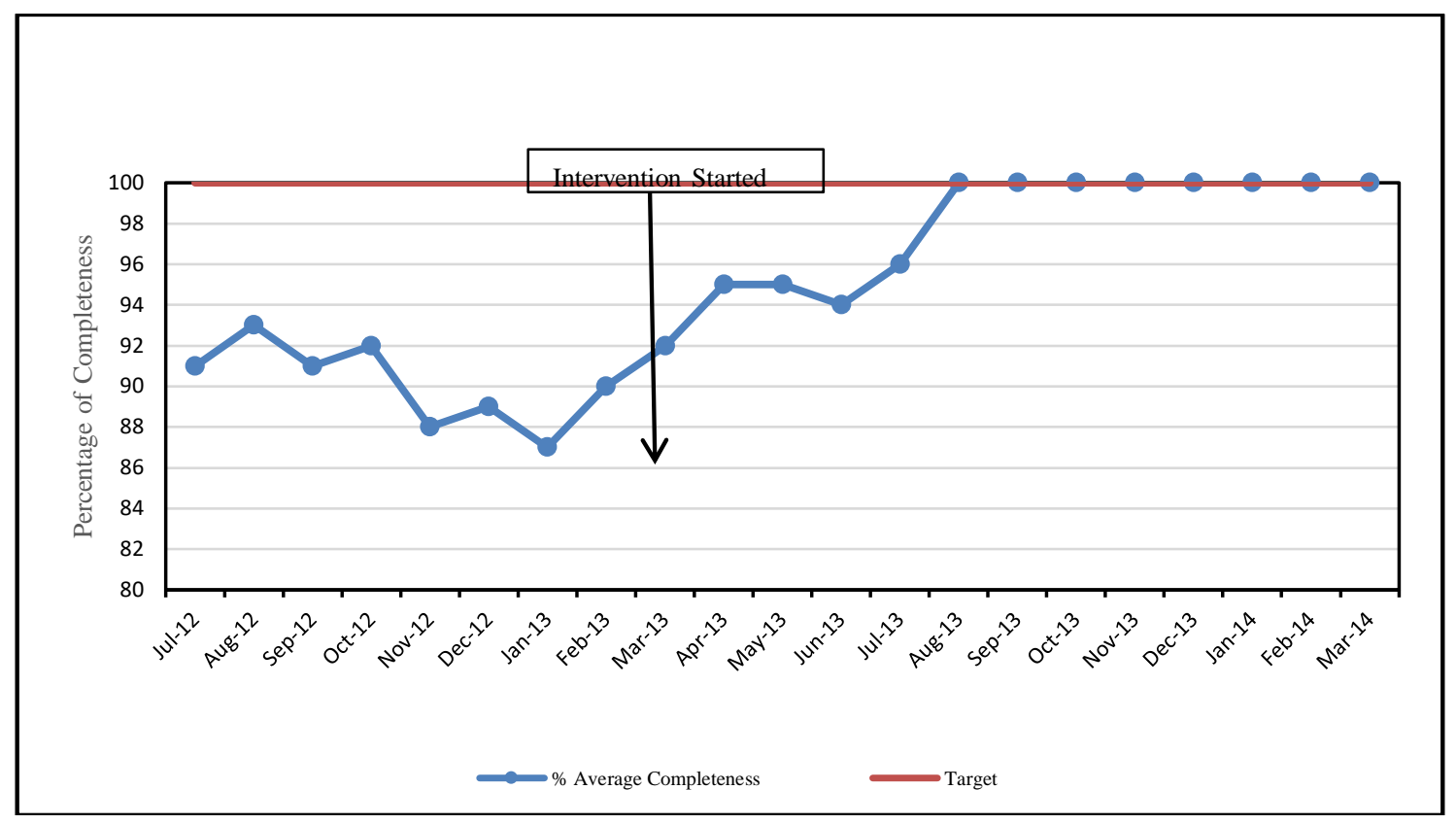

Figure 2. Percentage average data completeness of monthly midwifery returns

\subsection{Support supervision and feedback}

Table 3 shows the performance of supervision and feedback from supervisors. As indicated in Table 3, the overall level of supervision performance was 15 percent in baseline and 90 percent in end line. However, 50 percent and 100 percent in baseline and end line respectively of the facilities reported that sub-municipal/municipal level supervisors visited their facilities once during the last three months prior to the surveys. Twenty percent and 100 percent of the facilities in baseline and end line respectively reported that the supervisors performed some data quality checks during supervisory visits. Similar percentages also indicated that the municipal/sub-municipal supervisor discussed performance of the health facility based on RHIS information when the visit was made. Some 10 percent and 85 percent of facilities in baseline and end line respectively reported that the supervisors helped them make decisions based on the RHIS information.

Table 3. Supervision and feedback

\begin{tabular}{|c|c|c|c|c|}
\hline \multirow{2}{*}{$\begin{array}{l}\text { Supervision by the municipal/sub-municipal health } \\
\text { office }\end{array}$} & \multicolumn{2}{|c|}{ Baseline (2012) } & \multicolumn{2}{|c|}{ End line (2014) } \\
\hline & Yes & No & Yes & No \\
\hline $\begin{array}{l}\text { Did the municipal/sub-municipal supervisor visit } \\
\text { your facility at least once during the last three } \\
\text { months? (check the answer) }\end{array}$ & $\begin{array}{c}10 \\
(50 \%)\end{array}$ & $\begin{array}{c}10 \\
(50 \%)\end{array}$ & $\begin{array}{c}20 \\
(100 \%)\end{array}$ & $\begin{array}{c}0 \\
(0 \%)\end{array}$ \\
\hline $\begin{array}{l}\text { Did you observe supervisor having a checklist to } \\
\text { assess the data quality? }\end{array}$ & $\begin{array}{c}0 \\
(0 \%)\end{array}$ & $\begin{array}{c}20 \\
(100 \%)\end{array}$ & $17(85 \%)$ & $\begin{array}{c}3 \\
(15 \%)\end{array}$ \\
\hline Did supervisor check the data quality? & $\begin{array}{c}4 \\
(20 \%)\end{array}$ & $\begin{array}{c}16 \\
(80 \%)\end{array}$ & $\begin{array}{c}20 \\
(100 \%)\end{array}$ & $\begin{array}{c}0 \\
(0 \%)\end{array}$ \\
\hline $\begin{array}{l}\text { Did the municipal/sub-municipal supervisor discuss } \\
\text { performance of health facilities based on RHIS } \\
\text { information when he/she visited your facility? }\end{array}$ & $\begin{array}{c}4 \\
(20 \%)\end{array}$ & $\begin{array}{c}16 \\
(80 \%)\end{array}$ & $\begin{array}{c}20 \\
(100 \%)\end{array}$ & $\begin{array}{c}0 \\
(0 \%)\end{array}$ \\
\hline $\begin{array}{l}\text { Did the supervisor send a report/feedback/note on } \\
\text { the last two supervisory visits? }\end{array}$ & $\begin{array}{c}0 \\
(0 \%)\end{array}$ & $\begin{array}{c}20 \\
(100 \%)\end{array}$ & $16(80 \%)$ & $\begin{array}{c}4 \\
(20 \%)\end{array}$ \\
\hline Overall level of Supervision Performance & $\begin{array}{c}3 \\
(15 \%)\end{array}$ & $\begin{array}{c}17 \\
(85)\end{array}$ & $18(90 \%)$ & $\begin{array}{c}2 \\
(10 \%)\end{array}$ \\
\hline
\end{tabular}

Nonetheless, all the facilities indicated that none of the supervisors had a checklist to assess data quality in baseline but in end line, 80 percent of the facilities indicated that a supervisor came along with checklist. Also baseline results indicated that none of the supervisors sent a report or feedback to the facility on the previous two supervisory visits. However, the situation has improved in end line as 80 percent of the facilities confirmed that supervisors sent them feedback after each supervisory visit as shown in Table 3. 


\subsection{Data quality: completeness and accuracy}

Data quality refers to the condition of a set of values of qualitative or quantitative variables. There are many definitions of data quality but data is generally considered as high quality if it is fit for its intended uses in operation, planning and decision-making. Data quality is inextricably linked to the use of Information Systems and the health sector is increasingly an information-driven service [23]. The quality of generated, routinely and non-routinely in the health sector and stored, in whatever method is critical to the health and well-being of patients, the wider population, and to the management of health care organizations [24]. Raising the level of data quality within an organization contributes to improving the quality of decision-making enabling the reduction of uncertainty and the production of more timely and accurate decision outcomes.

Previous studies estimated that up to $5 \%$ of data found in organizations are of poor quality [25] and that the average perceived cost of poor data quality is as high as $10 \%$ of an organization's revenues [26]. In the healthcare sector, lack of data quality has far-reaching effects. Planning and delivery of services rely heavily on data from clinical, administrative and management sources [27]. For example, evidence-based practice [28] requires access to extensive research data, collated and presented in a way that a clinician can use at the time of diagnosis or in other decision-making situations. The higher the quality of the data, the better will be the patient outcomes. Likewise, quality data, particularly with regard to timeliness and accuracy, are needed for administrative purposes such as hospital bed-rostering and for planning services to ensure that they are cost-effective. These different but interlocking data requirements and decisions ensure that health care organizations and their relationships are inherently complex and demanding [29].

Poor quality data leave room for proxy measurement which is based on assumption. This affects the quality of planning and decision-making that uses the proxy indicators provided, especially when the assumption(s) fail. For that matter, it is imperative to have quality datasets that represent the realities on the ground. Thus, the improvement in the accuracy and completeness will lead to high quality and reliable RHIS for planning and decision-making at facility, management and policy level with high level of accuracy and confidence. The result indicates improvement in the overall data accuracy and completeness. For example, there was improvement in data accuracy and completeness from 4 out of 20 facilities in baseline to a little over 18 out of 20 in end line as shown in Table 1 and Table 2. The end line study shows that 18 out of 20 facilities report all the required number of the RHIS monthly reports to the District Health Information Management System (this excludes the number of data items for services not provided by this health facility) timely. The outstanding performance of the quality of data generated at the Ejisu-Juaben Municipal Health Service conformed to Lippeveld and colleagues' [30] definition which described data quality in four dimensions: relevance, completeness, timeliness and accuracy. However, this study did not assess the relevance of information generated at the municipal health directorate because all the information generated are requirements of the national health information system which is determined by the Policy Planning, Monitoring and Evaluation (PPME) unit of the Ghana Health Service.

\subsection{Data accuracy and completeness impact}

Mentoring and coaching of frontline providers in data quality improvement process, with quarterly data reconstruction at the facility level, coupled with regular feedback are ingredients for improving data quality contributed to improved Antenatal data accuracy and completeness in midwifery data as shown in Figure 1 and Figure 2]. Specific change ideas tested and implemented such as the use of provisional registers and tally sheets instead of loose papers for documenting community outreach services rendered at the community away from the health facility. This approach addressed issue of service providers been unable to carry along bulky registers during community outreach services, thereby committing errors and omissions during transcribing into the official registers later. Other change ideas implemented include training update Maternal and Child Health indicators definitions. Persistent and reliable implementation of these strategies coupled with commitment of management and frontline providers impacted continuous improvement in data quality as shown in Figure 1 and Figure 2.

\subsection{Support supervision and feedback}

The performance of support supervision by the Municipal/Sub-municipal supervisors is measured by number of supervisory visit to the facility at least once in a quarter, and supervisors having checklists to assess data quality and use of information. The supervisors are also supposed to discuss performance of the health facilities based on RHIS information whenever he/she visited the facility. The supervision indicator also measured supervisor's assistance to the facility to make a decision based on RHIS information, and supervisor sending a report/feedback/note to the facility on the last two supervisory visits. In baseline 3 out of 20 facilities received supervisory visits compared to 18 out of 20 in the end line as shown in Table 3 . This spectacular performance might have impact on improvement in data accuracy and completeness [31-33]. 


\subsection{Limitations of the study}

While this design allows the study to document changes in outcome indicators in target beneficiary the intervention; it is difficult to know to what extent these changes are actually due to this study. Extraneous factors could have either a positive or negative effect on study's intended outcomes, thus hiding the programs true effect.

\section{CONCLUSION}

Training, monitoring and provision of regular feedback to frontline staff in data quality improvement enhance data accuracy and completeness. This has implications for decision-making and resource allocation, especially in an era of new orientation and growing interest towards quality data, and in low-income countries, where the routine health information management system relies heavily on paper work.

\section{ACKNOWLEDGEMENTS}

The Authors are grateful to study participants and the Ejisu Juaben Municipal Health Team for their contributions to the successful completion of the study. We appreciate the support of the staff of School of Public Health, Kwame Nkrumah University of Science and Technology

\section{REFERENCES}

[1] Mavimbe JC, Braa J, Bjune G. Assessing immunization data quality from routine reports in Mozambique. $B M C$ Public Health, 11:108. [PMC free article] [PubMed], 2005.

[2] Odhiambo-Otieno GW. Evaluation of existing district health management information systems a case study of the district health systems in Kenya. International Journal of Medical Informatics, 74:733-44. [PubMed], 2005b

[3] Sprague C., Chersich M. F., Black, V. Health system weaknesses constrain access to PMTCT and maternal HIV services in South Africa: a qualitative enquiry. AIDS Research and Therapy 8:10, 2011

[4] MOH. A Bulletin of Health Information: Information for Action. Volume 1, Number 1. Accra, Ghana. p. 1 July September, 2001.

[5] Arah O. A. , Klazinga N. S., Delnoij D. M. J., Ten Asbroek A. H. A., Custers T. Conceptual frameworks for health systems performance: a quest for effectiveness, quality, and improvement. International Journal for Quality in Health Care, Volume 15, Issue 5, Pages 377-398, 1 October, 2003

[6] Srinath R, Vikram P, Prabhat J, Vinod K. P, Shiva K, Lalit D. Towards achievement of universal health care in India by 2020: A call to action. The Lancet India Group for Universal Healthcare. Volume 377, Issue 9767, Pages 760-768, 26 February-4 March, 2011

[7] WHO. Mechanisms to address the low understanding and unreliably and untimely information include facilitative supervision, on-the-job training, bi-annual and annual performance hearing, 2000.

[8] MOH, (2007). HMIS Assessment using HMN Assessment Tool

[9] Lippeveld T, Sauerborn R, Bodart C. Design and implementation of health information systems. Geneva: World Health Organization. 2000.

[10] Aqil A, Lippeveld T, Hozumi D: PRISM framework: a paradigm shift for designing, strengthening and evaluating routine health information systems. Health Policy and Planning, 24:217-228, 2009

[11] Chen H, Hailey D, Wang N and Yu P. A Review of Data Quality Assessment Methods for Public Health Information Systems. Int. J. Environ. Res. Public Health 11(5), 5170-5207; 2014

[12] Powell A E, Davies H T O, Thomson R G. Using routine comparative data to assess the quality of health care: understanding and avoiding common pitfalls. Qual Saf Health Care, 12:122-128, 2003.

[13] Karolyn K, Tony N, Rosemary S. Data Quality Information and Decision Making, 2007

[14] Davies HT, Crombie IK. Interpreting health outcomes. J Eval Clin Pract, 3:187-99, 1997.

[15] Iezzoni LI. Using risk-adjusted outcomes to assess clinical practice: an overview of issues pertaining to risk adjustment. Ann Thorac Surg, 58:1822-6.

[16] Black N. High-quality clinical databases: breaking down barriers. Lancet, 353:1205-6, 1999.

[17] Hozumi D, Aqil A, Lippeveld T. Pakistan situation analysis. MEASURE Evaluation Project, USAID, 2002.

[18] JICA HMIS Study Team. Assessment of health information systems in the health sector in Pakistan. Scientific System Consultants (Japan), Pakistan Ministry of Health, JICA, 2004.

[19] Aqil A. UPHOLD Project. Kampala: John Snow, Inc. (JSI); HMIS and EMIS situation analysis, Uganda, 2004.

[20] Aqil A, Lippeveld T. Improving RHIS Performance for Better Health System Management, Trainer's guide, MEASURE Evaluation, USAID, 2008. 
[21] Aqil A, Lippeveld T, Yokoyama R. Guangxi Baseline HMIS Report. MEASURE Evaluation, CDC Guangxi, USAID, 2007b.

[22] Ghana Health Service (GHS), Family Health Division Annual Report, 2015 (https://www.ghanahealthservice.org/downloads/2015_FAMILY_HEALTH_DIVISION_ANNUAL_REPORT.pdf)

[23] Hovenga, E. J. S., Kidd, M. R., \& Cesnik, B. Health Informatics. An Overview. Melbourne: Churchill Livingstone, 1996.

[24] Long, J. A., \& Seko, C. E. A new method for database data quality evaluation at the Canadian Institute for Health Information, 7th International Conference on Information Quality. Boston: Massachusetts Institute of Technology, 2002.

[25] Redman, T. C. Data Quality. The Field Guide. Boston: Digital Press, 2001.

[26] Nsubuga P, Eseko N, Tadesse W, et al. (2002). Structure and performance of infectious disease surveillance and response, United Republic of Tanzania, Bulletin of the World Health Organization, 80:196-203. [PMC free article] [PubMed], 1998

[27] Karolyn K, Tony N, Rosemary S. Data Quality Information and Decision Making: A Healthcare Case Study. 18th Australasian Conference on Information Systems Data Quality in Healthcare 5-7, Toowoomba, Dec 2007

[28] Gendron, M. S., \& D'Onofrio, M. J. Data Quality in the Healthcare Industry. Data Quality Journal, vol 7, no. $1,2001$.

[29] Lippeveld T, Sauerborn R, Bodart C. Design and implementation of health information systems. Geneva: World Health Organization, 2000.

[30] Perloff RM. The dynamics of persuasion. Hillsdale, NJ: Lawrence Erlbaum Associates, 1993.

[31] Odhiambo-Otieno GW. Evaluation of existing district health management information systems a case study of the district health systems in Kenya. International Journal of Medical Informatics, 74:733-44. [PubMed], 2005b.

[32] Rotich JK, Hannan TJ, Smith FE, et al. (2003) Installing and implementing a computer-based patient record system in sub-Saharan Africa: The Mosoriot Medical Record System. Journal of the American Medical Informatics Association.;10:295- 303. [PMC free article] [PubMed], 2003

[33] A Healthcare Case Study. 18th Australasian Conference on Information Systems Data Quality in Healthcare 5-7, Toowoomba, Dec 2007.

\section{BIOGRAPHIES OF AUTHORS}

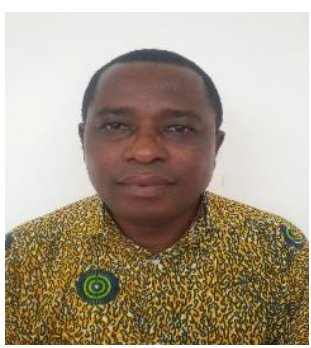

Richard Okyere Boadu, $\mathrm{PhD}$, is a lecturer, research and consultancy coordinator at the Department of Health Information Management in University of Cape Coast of Ghana. Prior to his current appointment, he worked with various international NGOs and Ghana Catholic Health Service as Strategic Information Monitoring \& Evaluation Advisor. He has a BSc. in Computer Science, an MSc. in Health Services Planning and Management and a PhD in Public Health, all from the Kwame Nkrumah University of Science and Technology in Kumasi, Ghana as well as a diploma in Management Information Systems from the Ghana Institute of Management and Public Administration. He is also an IHI-trained, certified and practicing Improvement Advisor.

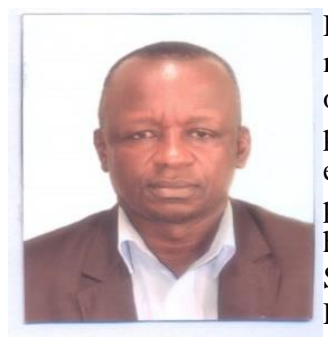

Dr. Peter Agyei-Baffour is a Public Health Specialist with affection for health policy, management and finance. Using a transdisciplinary and multidisciplinary approaches. The goals of my teaching, research and service are to take policy, management, financing and economics perspectives in analyzing health systems to guide the formulation and implementation of evidence-based and evidence-informed policies, and to use knowledge broker platforms such as policy dialogues, conferences and publications to share, discuss and solicit inputs to address health systems challenges to facilitate the realization of the Universal Health Coverage and other Sustainable Development Goals. I hold PhD in Public Health, MA in Industrial Management and Postdoctoral Certificate in Global Health and currently, Head, Department of Health Policy Management and Economics, School of Public Health, Kwame Nkrumah University of Sciences and Technology, Kumasi, Ghana 


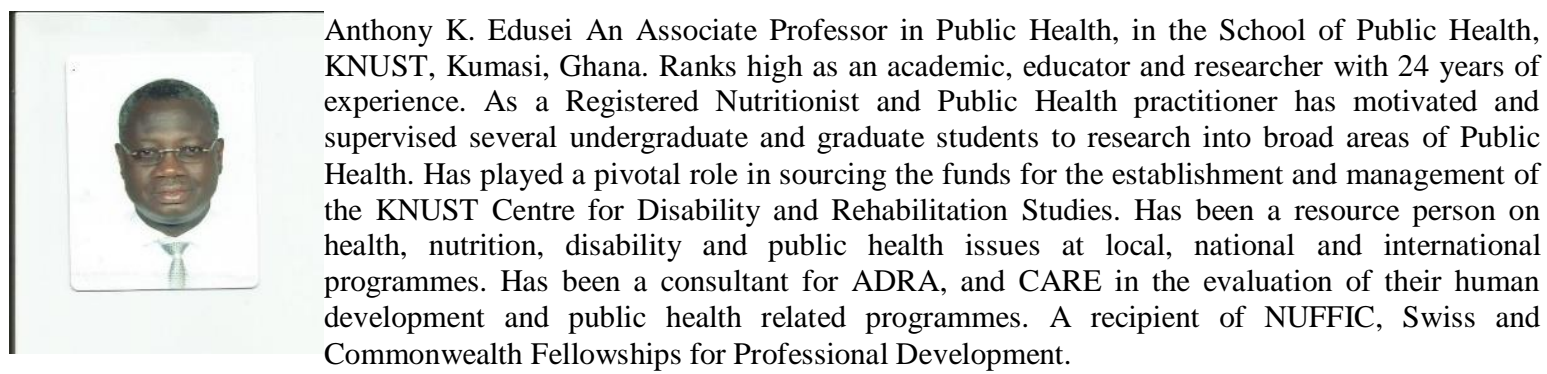

Anthony K. Edusei An Associate Professor in Public Health, in the School of Public Health, KNUST, Kumasi, Ghana. Ranks high as an academic, educator and researcher with 24 years of As a Registered Nutritionist and Public Health practitioner has motivated and he KNUST Centre for Disability and Rehabilitation Studies. Has been a resource person on health, nutrition, disability and public health issues at local, national and international development and public health related programmes. A recipient of NUFFIC, Swiss and Commonwealth Fellowships for Professional Development. 